\title{
REGULAR AND BAER RINGS
}

\author{
K. M. RANGASWAMY
}

\begin{abstract}
The (von Neumann) regular Baer rings representable as the full ring $E(G)$ of all endomorphisms of an abelian group $G$ are characterized. It is also shown that a countable regular Baer ring is Artinian semisimple.
\end{abstract}

All the rings that we consider are associative with identity and all the modules are unitary left modules. A ring $R$ is called (von Neumann) regular if each $a \in R$ satisfies $a=a x a$ for some $x \in R$. $R$ is strongly regular if each $a$ satisfies $a=x a^{2}=a^{2} x$ for some $x \in R$. A ring $R$ is said to be Baer if every left (equivalently, right) annihilator ideal is generated by an idempotent [3]. The additive group of a ring $R$ is denoted by $R^{+}$. Let $G$ be an abelian group. Then $E(G)$ will denote the ring of all endomorphisms of $G, G_{t}$ the subgroup of all elements of finite order and, for each prime $p$, $G_{p}$ the subgroup of all elements of order a power of $p$. A subgroup $S$ is pure in $G$ if $n S=S \cap n G$ for every positive integer $n$. A subring $S$ is pure in $R$ if $S^{+}$is pure in $R^{+}$. If $\left\{A_{i}\right\}, i \in I$, are rings or groups, then $\prod_{i \in I} A_{i}$ $\left(\oplus_{i \in I} A_{i}\right)$ denotes their direct product (sum). $Q$ denotes the field of rational numbers and $Z(p)$ the field of integers modulo a prime $p$.

1. Strongly regular Baer rings. First note that a regular ring is strongly regular if and only if it has no nonzero nilpotent elements or, equivalently, all its idempotents are central. Thus for a strongly regular ring $R$ the lattice $L(R)$ of all principal left ideals of $R$ is isomorphic to the Boolean algebra $B(R)$ of all idempotents in $R$. Moreover the lattice of all left (=right) ideals of $R$ is isomorphic to the lattice of all ideals of $B(R)$. Hence from a well-known theorem of $\mathrm{M}$. H. Stone we get: A strongly regular ring $R$ is Baer $\Leftrightarrow B(R)$ is complete $\Leftrightarrow$ the maximal ideal space of $R$ is extremely disconnected. Since a complete Boolean algebra cannot be countably infinite, the last remark implies that a countable strongly regular Baer ring is Artinian semisimple. Actually a more general result holds, as indicated below.

Received by the editors January 5, 1972 and, in revised form, May 13, 1973.

AMS (MOS) subject classifications (1970). Primary 20K30, 16A30, 16A34.

Key words and phrases. Abelian groups, endomorphism rings, von Neumann regular rings, Baer rings, strongly regular rings.

(c) American Mathematical Society 1974 
THEOREM 1. A regular Baer ring of cardinality $<c$, the continuum is Artinian semisimple.

Proof. It is enough if we show that $R$ satisfies the A.C.C. for principal left ideals. Suppose $R e_{1} \subset R e_{2} \subset \cdots$ is a strictly increasing chain where $e_{n}=e_{n}^{2}$ for all $n$. Clearly $e_{n} e_{n+1}=e_{n}$ for all $n$. Define inductively $f_{1}=e_{1}, f_{n+1}=f_{n}+e_{n+1}-e_{n+1} f_{n}$. It is then easy to check that $R e_{n}=R f_{n}$ and $f_{n} f_{n+1}=f_{n+1} f_{n}=f_{n}$ for all $n$. Hence, by induction, $f_{n} f_{m}=f_{m} f_{n}=f_{n}$ for all $m>n$. Define $g_{n}=f_{n}-f_{n+1}$. Then $g_{n}^{2}=g_{n}$ for all $n$ and $g_{m} g_{n}=0$ if $m \neq n$. Note that $g_{n} \neq 0$, since $R f_{n}=R e_{n} \neq R e_{n+1}=R f_{n+1}$. To each nonempty subset $S$ of natural numbers, choose an idempotent $h_{S}$ in $R$ such that the right annihilator of $\left\{g_{i}: i \in S\right\}$ is $\left(1-h_{S}\right) R$. Clearly $g_{i} h_{S}=g_{i}$ for all $i \in S$, and $\left(1-h_{S}\right) g_{k}=g_{k}$ (i.e., $\left.h_{S} g_{k}=0\right)$ for all $k \notin S$. Note that $h_{S} g_{k}=0$ implies $g_{k} h_{S} g_{k}=0$ so that $g_{k} h_{S} \neq g_{k}$. Hence if $S$ and $T$ are distinct subsets of natural numbers, then $h_{S} \neq h_{T}$. This contradicts the hypothesis that $R$ has cardinality less then $c$. Hence the result.

2. Regular and Baer endomorphism rings. In this section we completely characterize those regular Baer rings which appear as the full rings of endomorphisms of abelian groups. These rings turn out to be 'almost' self-injective. Since our work here is a continuation of [6], we freely make use of the results and terminology of [6].

LEMMA 1. Let $S$ be a pure subgroup of $\Pi Z(p)^{+}$, where $p$ runs over a set of primes, such that $\oplus Z(p)^{+} \subseteq S \subseteq \prod Z(p)^{+}$. Then the following are true:

(i) if $\rho: S \rightarrow S$ is a Z-morphism then, for any element $x=\left\langle\cdots, x_{p}, \cdots\right\rangle$ in $S, x \rho=\left\langle\cdots, x_{p} \rho_{p}, \cdots\right\rangle$ where $\rho_{p}=\rho \mid Z(p)$;

(ii) every endomorphism of $S$ uniquely extends to an endomorphism of $\prod Z(p)^{+}$.

LeMma 2. Let $P$ be a set of primes and $R$ a pure subring of $\prod_{n \in P} Z(p)$ containing $\oplus Z(p)$ and the identity. Then $R \cong \operatorname{Hom}_{Z}\left(R^{+}, R^{+}\right)$.

Proof. Since $R \cong \mathrm{Hom}_{R}(R, R)$, it is enough to show that every $Z$-endomorphism of $R$ is an $R$-morphism. But this is immediate from Lemma 1(i) and the fact that, for all $p \in P, \rho_{p}=\rho \mid Z(p)$ is an $R$-morphism.

As a corollary we get a description of strongly regular endomorphism rings (see Problems 44 and 50 in [1]).

COROLlary. Let $R$ be strongly regular. Then $R=E(G)$ for some abelian group $G$ if and only if either $R \cong \prod F_{i}$, where the $F_{i}$ are nonisomorphic prime fields or $R$ is a regular subring with identity of $\prod_{p \in I^{\prime}} Z(p)$ containing $\oplus Z(p)$, where $P$ is a set of primes. 
Proof. $R=E(G)$ regular implies (see [5]) that either $G=D \oplus E$, where $D$ is a direct sum of copies of $Q^{+}$and $E=\oplus E_{p}$, each $E_{p}$ being a direct sum of copies of $Z(p)^{+}$or $G$ is a pure subgroup of $\prod G_{p}$ and each $G_{p}$ is a direct sum of copies of $Z(p)^{+}$. If $G$ contains a summand of the form $A \oplus A$, then it is easy to construct an endomorphism $\rho \neq 0$ such that $\rho^{2}=0$. Since $R$ has no nonzero nilpotent elements, we get, in the first case, that $D \cong Q^{+}$and $E_{p} \cong Z(p)^{+}$and, in the second case, that $G_{p}=Z(p)^{+}$. This proves the necessity. The sufficiency follows from Lemma 2 if one notes that a regular subring of $\prod Z(p)$ is pure.

We are now ready to prove the main theorem of this paper.

THEOREM 2. Let $R$ be a regular Baer ring. Then $R \cong E(G)$ for some abelian group $G$ if and only if $R$ is a ring direct sum, $R=R_{\mathbf{1}} \oplus R_{\mathbf{2}}$, where

(i) $R_{2}$ is left self-injective being isomorphic to $\prod_{i \in N} L_{i}$ where, for each $i \in N, L_{i} \cong \operatorname{Hom}_{F_{i}}\left(V_{i}, V_{i}\right)$ with $V_{i}$ a vector space of dimension $\geqq 2$ over a prime field $F_{i}$ and $F_{i} \not F_{j}$ if $i \neq j$, and

(ii) $R_{1}$ is a (commutative) regular subring containing all the idempotents of the ring $\prod_{p \in T} Z(p)$, where $T$ is a set of primes and no $p$ in $T$ is the characteristic of any of the fields $F_{i}$. Moreover, $R_{1}=0$ if any of the fields $F_{i}$ has characteristic zero.

Proof. Suppose $R \cong E(G)$ for some abelian group $G$. Then, by Remark 4.2 of [6], either (a) $G=D \oplus S, D$ torsion-free divisible and $S=\oplus S_{p}$ is elementary, $p$ running over a set of primes, or (b) $G$ is reduced, $G_{t}$ is elementary and $G / G_{t}$ is divisible. In the case (a), $D$ and $S_{p}$ can be considered as vector spaces over the fields $Q$ and $Z(p)$ respectively and their $Z$-endomorphisms are vector space morphisms. Consequently,

$$
R \cong \operatorname{Hom}_{Q}(D, D) \oplus \prod_{p} \operatorname{Hom}_{Z(p)}\left(S_{p}, S_{p}\right)
$$

which is in the required form. Consider the case (b). We can write $\oplus G_{p} \subseteq$ $G \subseteq \prod G_{p}$. Since every endomorphism of $G$ uniquely extends to an endomorphism of $\prod G_{p}$, we consider $R=E(G)$ as a subring of $E\left(\prod G_{p}\right) \cong$ $\prod E\left(G_{p}\right)$ containing $\oplus E\left(G_{p}\right)$. Let $T$ be the set of all primes $p$ for which $G_{p}$ is cyclic. Define $S_{1}=\bigoplus_{p \in T} G_{p}$ and $S_{2}=\bigoplus_{p \notin T} G_{p}$. If $A_{i}(i=1,2)$ is the closure of $S_{i}$ under the $n$-adic topology of $G$, then, by Theorem 3.3 of [6], $G=A_{1} \oplus A_{2}$. Clearly the $A_{i}$ are invariant under all the endomorphisms of $G$ so that $R \cong E\left(A_{1}\right) \oplus E\left(A_{2}\right)$.

Let $R_{1}=E\left(A_{1}\right)$. Since $\left(A_{1}\right)_{p}=Z(p)^{+}, R_{1}$ is isomorphic to a subring of $\prod_{p \in T} Z(p)$ so that $R_{1}$ is commutative. Since $R_{1}$ is Baer, by Theorem 3.3 and Remark 3.5 of [6], $R_{1}$ contains all the idempotents of $\prod_{p \in T} Z(p)$.

Let $R_{2}=E\left(A_{2}\right)$. Now $R_{2}$ is regular and Baer and hence is left continuous, by [6]. Let $I$ be a nonzero two sided ideal of $R_{2}$. We wish to show that $I$ 
contains a nonzero nilpotent element. Since $\left(R_{2}\right)_{p} \cong E\left(G_{p}\right)$ and $\bigoplus\left(R_{2}\right)_{p} \subseteq$ $R_{2} \subseteq \prod\left(R_{2}\right)_{p}$, and since each $\left(R_{2}\right)_{p}$ is an elementary abelian p-group, $I$ contains a nonzero $\rho$ satisfying $p \rho=0$ for some prime $p$. Then $\operatorname{Im} \rho \subset$ the elementary abelian $p$-group $G_{p}$ and, composing $\rho$ with a projection of $G_{p}$ onto a cyclic summand of $G_{p}$, get a $\lambda \in I$ with $\operatorname{Im} \lambda$ a cyclic summand, say $G=\operatorname{Im} \lambda \oplus C$. If $\operatorname{ker} \lambda \cap G_{p}=0$, then $G_{p}$ would isomorphically map into $\operatorname{Im} \lambda$ and hence $G_{p}$ is cyclic, a contradiction. Thus $\operatorname{ker} \lambda \cap G_{p} \neq 0$. Let $\mu$ be an endomorphism of $G$ given by $\mu \mid C=0$ and $(\mu \mid \operatorname{Im} \lambda)=a$ monomorphism from $\operatorname{Im} \lambda$ to $\left(G_{p} \cap \operatorname{ker} \lambda\right)$. Then $0 \neq \lambda \mu \in I$ and $(\lambda \mu)^{2}=0$. Thus every nonzero two sided ideal of the left continuous regular ring $R_{2}$ contains a nonzero nilpotent element. By Theorem 3 of [7], $R_{2}$ is therefore left self-injective. Then Theorem 4.6 of [6] implies that $R_{2} \cong \prod_{i \in N} L_{i}$ with $L_{i} \cong \operatorname{Hom}_{F_{i}}\left(V_{i}, V_{i}\right)$, where the $F_{i}$ 's are prime fields with nonzero characteristic and $F_{i} \not F_{j}$ if $i \neq j$.

Thus in both cases, $R=R_{1} \oplus R_{2}$, where the $R_{i}$ 's have the required form. Moreover, $R_{1} \neq 0$ only in the case (b) wherein none of the $F_{i}$ has characteristic zero.

Conversely, let $R=R_{1} \oplus R_{2}$ be in the required form. Since the $F_{i}$ are prime fields, $\operatorname{Hom}_{F_{i}}\left(V_{i}, V_{i}\right)=\operatorname{Hom}_{Z}\left(V_{i}, V_{i}\right)$ and since the $F_{i}$ are nonisomorphic, $R_{2} \cong E(H)$ where $H=\bigoplus V_{i}^{+}$. Now by Lemma $2, R_{1}=E\left(R_{1}^{+}\right)$. If $R_{1} \neq 0$, then each $F_{i}$ has, by hypothesis, characteristic $p_{i}>0$ so that, in view of our assumption on the set $T$,

$$
\operatorname{Hom}_{Z}\left(R_{1}^{+}, H\right)=0=\operatorname{Hom}_{Z}\left(H, R_{1}^{+}\right) .
$$

Then $R=E(G)$, where $G=H \oplus R_{1}^{+}$.

REMARK. If $R$ is regular Baer and $R \cong E(G)$, then $R$ need not be selfinjective. This is equivalent to saying that the ring $R_{1}$ of Theorem 2 need not be self-injective. To see this, let $P$ be the set of all prime integers $>0$ and let $A$ be the subring of $\prod_{p \in P} Z(p)$ containing $\oplus Z(p)$ and the identity such that $A /(\oplus Z(p)) \cong Q$, the field of rational numbers (see [2]). Clearly $A$ is regular. Let $B$ be the subring generated by $A$ and all idempotents of $\Pi Z(p)$. Since $B=\sum A e$, where $e$ runs over all the idempotents of $\prod Z(p)$, it is easy to see that $B$ is regular (and Baer). Now $B \neq \Pi Z(p)$, since $B$ does not contain the element $x=\left\langle\cdots, x_{p}, \cdots\right\rangle$, where $x_{p}^{2} \equiv$ $-1(\bmod p)$, if $p$ is of the form $4 n+1$ and $x_{p}=0$, otherwise. [Note that, to each $y \in A$, there exists a rational number $s / t$ with $(s, t)=1$ and $t y_{p} \equiv$ $s(\bmod p)$ for all except a finite number of primes $p \in P$.] Since $B$ is distinct from its injective envelope $\Pi Z(p), B$ is not self-injective.

By remark 4.3 of [6], we have the following

COROllaRY. Let $G$ be an abelian group. Then $E(G)$ is regular Baer if and only if either $G=D \oplus E$, where $D \neq 0$ is torsion-free divisible and 
$E$ is elementary or $G$ is reduced and $G=A \oplus B$, where

(i) $A$ is a fully invariant subgroup of $\prod_{p \in P} E_{p}$ with $E_{p}$ being an elementary $p$-group and $P$ a set of primes, and

(ii) $B$ is a pure subgroup of $\prod_{p \in P} Z(p)^{+}$with the property that every endomorphic image and also each subgroup closed under the n-adic topology of $B$ is a direct summand of $B$, where $P^{\prime}$ is another set of primes with $P \cap P^{\prime}=\varnothing$.

\section{BIBLIOGRAPHY}

1. L. Fuchs, Abelian groups, Akad. Kiadó, Budapest, 1958; republished by Internat. Series of Monos. on Pure and Appl. Math., Pergamon Press, New York, 1960. MR 21 \#5672; 22 \#2644.

2. L. Fuchs and K. M. Rangaswamy, On generalized regular rings, Math. Z. 107 (1968), 71-81. MR 38 \#2171.

3. I. Kaplansky, Rings of operators, Benjamin, New York, 1968. MR 39 \#6092.

4. R. S. Pierce, Modules over commutative regular rings, Mem. Amer. Math. Soc. No. 70 (1967). MR 36 \#151.

5. K. M. Rangaswamy, Abelian groups with endomorphic images of special types, J. Algebra 6 (1967), 271-280. MR 36 \#271.

6. - Representing Baer rings as endomorphism rings, Math. Ann. 190 (1970/71), 167-176. MR 42 \#6105.

7. Y. Utumi, On continuous regular rings and semi-simple self-injective rings, Canad. J. Math. 12 (1960), 597-605. MR 22 \#8032.

Department of Mathematics, Institute of Advanced Studies, Australian National University, PO Box 4, Canberra, ACT 2600, Australia 